\title{
SPEED OBSERVER OF PERMANENT MAGNET SYNCHRONOUS BASED ON LEAST SQUARES SUPPORT VECTOR MACHINE REGRESSION
}

\author{
Muldi Yuhendri ${ }^{1 *}$, Hambali ${ }^{1}$, Mukhlidi Muskhir ${ }^{1}$ \\ ${ }^{1}$ Jurusan Teknik Elektro, Universitas Negeri Padang, Indonesia \\ Jl. Prof. Hamka Kampus UNP Air Tawar Padang \\ *Corresponding Author: muldiy@ft.unp.ac.id
}

\begin{abstract}
Motor speed control requires motor speed data as feedback of control actions. Motor speed data is usually obtained from the rotation speed sensor. This paper proposes the rotor speed observer for permanent magnet synchronous motor (PMSM) to obtain motor speed data based on back emf voltage. This method more economical without a rotation speed sensor. The rotor speed observer is designed by using the Model Reference Adapative System (MRAS) method with Least Squares Support Vector Machine Regression (LSSVMR) algorithm for adaptation mechanism. The proposed rotor speed observer is validated with varying motor speeds. The simualtion results show that the proposed MRAS observer by using LSSVMR algorithm as adaptation mechanism has successfully estimated the rotation speed of the PMSM based on the back emf motor voltage. It can be seen from the maximum error of tmotor speed, ie only $3.7 \mathrm{rpm}$ at transient conditions and close to zero at steady state.
\end{abstract}

Keywords: Permanent magnet motor, speed observer, MRAS, LSSVMR.

\section{INTRODUCTION}

Permanent magnet synchronous motor (PMSM) is one type of electric motor that is widely used to drive various types of machinery in the industry. This is because PMSM has many advantages compared to other types of motors, such as having high power density, high performance, high efficiency, easy to control and smaller size compared to other motor types for the same power [1]. The speed of the motor must be controlled according to the required engine speed. PMSM rotation speed control requires speed data and rotor position for the system of rotation speed control. The rotation speed and rotor position data are usually obtained from the rotor speed sensor. Therefore, in designing a PMSM rotation speed control system an additional cost is required for the procurement of a speed sensor. To reduce the cost of manufacturing a control system, a motor speed control system has been developed without a rotor speed sensor. This can be done by identifying the PMSM rotation speed based on other parameters, such as voltage, flux and stator current, also known as motor speed observer [2]. Several rotation speed observer methods have been developed for PMSM, such as kalman filter observer [3], flux observer and Model Reference Adaptive System (MRAS) observer [4] [5].

This paper proposes the MRAS observer to identify both PMSM speed and rotor position based on back emf voltage of motor. The advantages of MRAS obsrver are less complex, more effective than another observer methods and easier to implement [5]. MRAS observer consists of adaptive models, reference models and adaptation mechanisms [4]. The adaptation mechanism of the MRAS observer is usually implemented with various control methods, such as PI control, sliding mode and intelligent control system.

Several artificial intelligence algorithms that have been implemented for the adaptation mechanism of MRAS observer are fuzzy logic and neural network. In this paper, The adaptation mechanism of the MRAS obsever is proposed by using the Least Squares Support Vector Machine Regression (LSSVMR) method. LSSVMR is one type of machine learning with supervised training [6]. LSSVMR was chosen because it is global optimal in training data [7] - [9], so that it can increase validity and accelerate the work of the LSSVMR algorithm in 
identifying PMSM speed and rotor position based on the back emf voltage parameters.

\section{METHOD}

PMSM speed observer is proposed using MRS observer based on LSSVMR algorithm. In this method, the rotation speed and the rotor position of the PMSM are obtained by comparing the motor reference model with the adaptive model. The PMSM reference model is obtained from the actual parameters, while the adaptive model is obtained from the estimation parameter. LSSVMR is used as an adaptation mechanism between the adaptive model of PMSM and the reference model. The proposed MRAS observer was validated through simulations using Simulink Matlab. The LSSVMR model is designed by using the algorithm that has been discussed in [10]. Figure 1 shows the proposed speed observer scheme.

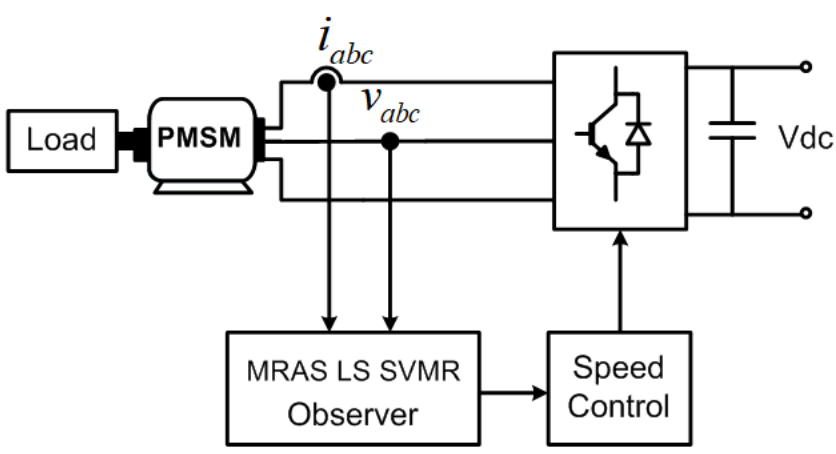

Figure 1. The proposed MRAS-LSSVMR observer scheme

The speed observer model shown in Figure 1 consists of a PMSM model that is connected to the load, current and voltage sensors, MRAS observer and LSSVMR algorithm as an adaptation mechanism, inverter and PMSM speed control.

\section{PMSM Model}

PMSM is an electric motor that uses permanent magnets to produce a rotating magnetic field [2]. MRAS observer design requires PMSM parameters in $\alpha \beta$ axis. The PMSM voltage equation in the $\alpha \beta$ axis is written as:

$$
\begin{aligned}
& V_{s \alpha}=-R_{s} i_{s \alpha}-L_{s} \frac{d i_{s \alpha}}{d t}+E_{\alpha} \\
& V_{s \beta}=-R_{s s \beta} i_{s \beta}-L_{s} \frac{d i_{s \beta}}{d t}+E_{\beta}
\end{aligned}
$$

Where $R_{s}, L_{s}$ are stator resistance and stator inductance, respectively. $V_{\alpha \beta}, i_{\alpha \beta}$ and $E_{\alpha \beta}$ are stator voltages, stator current and back emf voltage of PMSM in $\alpha \beta$ axis, respectively . Both stator currents and stator voltages $V_{\alpha b}, i_{\alpha \beta}$ can be obtained from three phase parameters by using $a b c-\alpha \beta$ transformation, that written as :

$$
\left[\begin{array}{l}
x_{\alpha} \\
x_{\beta}
\end{array}\right]=\frac{2}{3}\left[\begin{array}{rrr}
1 & -\frac{1}{2} & -\frac{1}{2} \\
0 & \frac{\sqrt{3}}{2} & -\frac{\sqrt{3}}{2}
\end{array}\right]\left[\begin{array}{l}
x_{a} \\
x_{b} \\
x_{c}
\end{array}\right]
$$

Where $\mathrm{x}$ can present the parameters of voltage, current, flux, back emf voltage and other PMSM parameters. The PMSM model for MRAS observers is presented in a state space equation, as shown in Figure 2.

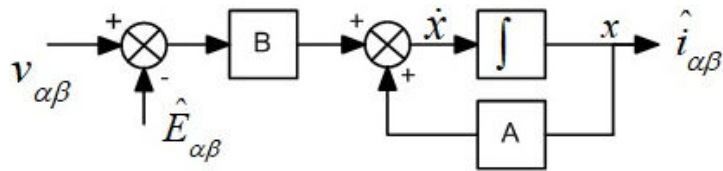

Figure 2. State space model of PMSM

The blocks A and B shown in Figure 2 are matrix of the stator coil resistance and the stator coil inductance, which are presented by the following equation:

$$
[A]=\left[\begin{array}{cc}
-R_{s} / L_{s} & 0 \\
0 & -R_{s} / L_{s}
\end{array}\right], \quad[B]=\left[\begin{array}{cc}
-1 / L_{s} & 0 \\
0 & -1 / L_{s}
\end{array}\right]
$$

\section{MRAS Observer}

The MRAS observer model for PMSM consists of the motor reference model, the motor adaptive model and the adaptation mechanism between the adaptive parameter and the reference parameter. The reference model presents the actual parameters of PMSM, while the adaptive model presents the motor parameters based on the estimation results of the adaptation mechanism [4]. The adaptation of the two models to the MRAS observer in terms of PMSM back emf voltage. Figure 3 shows the MRAS observer scheme for determining PMSM speed based on back emf voltage.

The estimated value of PMSM speed and rotor position in the MRAS observer are obtained from controlling the estimated back emf voltage to be the same as the reference back emf voltage. Regulating the estimated back emf voltage is done by adjusting the estimated stator current through an adaptation 
mechanism of observer. Adaptation mechanism can be done by various control methods, such as the Proportional Integral (PI) controller and several intelligent control methods [2]

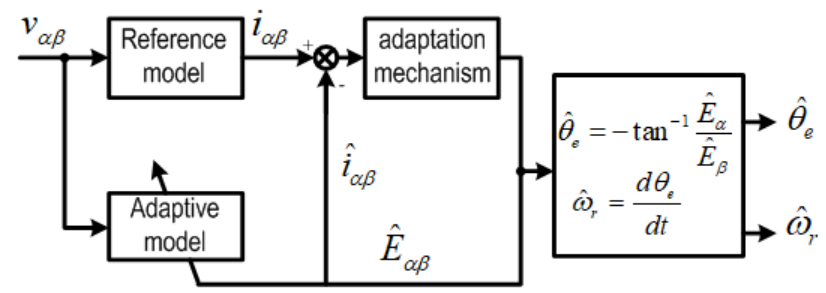

Figure 3. MRAS observer scheme

Based on Equations (1) and (2), the PMSM reference model for MRAS observers can be formulated as:

$\left[\begin{array}{c}\dot{i}_{\alpha} \\ \dot{i}_{\beta}\end{array}\right]=\left[\begin{array}{cc}-R_{s} / L_{s} & 0 \\ 0 & -R_{s} / L_{s}\end{array}\right]\left[\begin{array}{c}i_{\alpha} \\ i_{\beta}\end{array}\right]+\left[\begin{array}{cc}-1 / L_{s} & 0 \\ 0 & -1 / L_{s}\end{array}\right]\left(\left[\begin{array}{c}V_{\alpha} \\ V_{\beta}\end{array}\right]-\left[\begin{array}{c}E_{\alpha} \\ E_{\beta}\end{array}\right]\right)$

Based on the PMSM model in the state space equation shown in Figure 2 and based on reference model in Equation (5), the adaptive model of PMSM for MRAS observer can be written as:

$\left[\begin{array}{ll}\dot{i}_{\alpha} & \dot{\hat{i}}_{\beta}\end{array}\right]^{T}=[A]\left[\begin{array}{ll}\hat{i}_{\alpha} & \hat{i}_{\beta}\end{array}\right]^{T}+[B]\left(\left[\begin{array}{ll}V_{\alpha} & V_{\beta}\end{array}\right]^{T}-\left[\begin{array}{ll}\hat{E}_{\alpha} & \hat{E}_{\beta}\end{array}\right]^{T}\right)$

PMSM rotation speed as MRAS observer output is derived from the rotor position, which is obtained from the estimated back emf voltage using an adaptation mechanism. The estimated rotation speed $\omega_{r}$ and the estimated rotor position $\theta_{e}$ of the MRAS observer output can be formulated by :

$$
\begin{aligned}
& \hat{\theta}_{e}=-\tan ^{-1}\left(\hat{E}_{\alpha} / \hat{E}_{\beta}\right) \\
& \hat{\omega}_{r}=\frac{d \theta_{e}}{d t}
\end{aligned}
$$

The adaptation mechanism of observer MRAS is proposed using LSSVMR, as shown in Figure 4.The LSSVMR input is the reference current error and the estimated current from the adaptive model, while the output is the estimated back voltage emf which will determine the estimated PMSM speed based on Equation (7) and (8). The estimated current in the adaptive model is determined by the value of the motor voltage in the reference model and the value of the back voltage emf from the LSSVMR output. When the estimated current in the adaptive model is the same as the current in the reference model, the back voltage emf estimated by the LSSVMR will be the same as the emf back voltage in the reference model. In this condition, the estimated motor speed from the MRAS observer will be the same as the actual motor speed. Therefore, the validity of the motor speed observer results in MRAS is largely determined by the reliability of the adaptation mechanism in determining the estimated back voltage emf parameter, where this value will affect the estimated motor speed and the estimated current in the adaptive model.

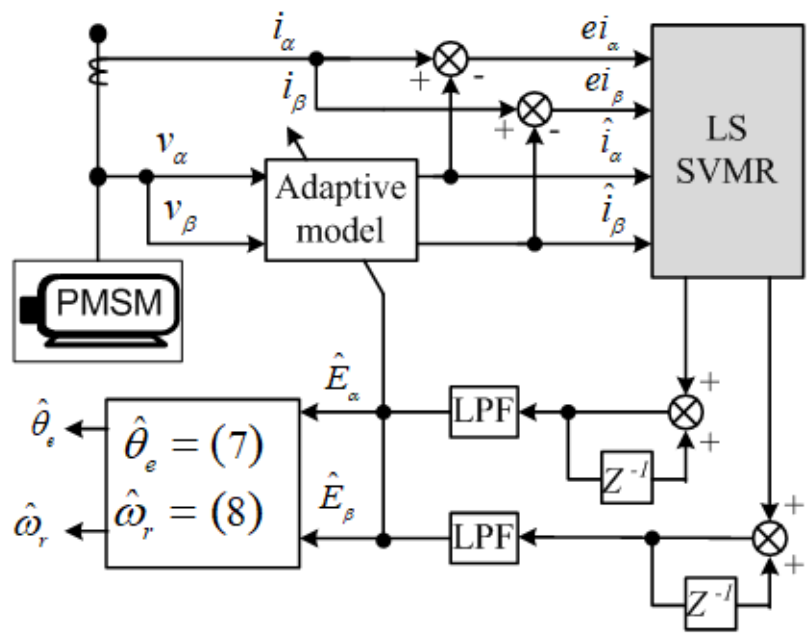

Gambar 4. The adaptation mechanism of MRAS observer based on LSSVMR

\section{Least Squares Support Vector Machine Regression}

Least Squares Support Vector Machine (LSSVM) is one of the learning machines with supervised learning methods that have global optimal characteristics [9]. LSSVM is an extension of the standard SVM. The difference between LSSVM and SVM is the optimization technique used. Standard SVM solves optimization problems with quadratic programming, while in LSSVM optimization problems are solved using linear equations, so it is faster in training data. LSSVM can be used for continuous data using LSSVM Regression (LSSVMR) or discrete data using LSSVM Classifier (LSSVMC) [10]. In this paper, LSSVMR was chosen, because the MRAS observer data to be processed was continuous.

The LSSVMR algorithm works by mapping nonlinear functions using linear functions to a higher dimensional space. If the LSSVM is used to train data with input $X$, output $y$ and a sample number $n$, then the LSSVMR regression function can be written formulated as:

$$
f(x, w)=w^{T} \varphi(x)+b
$$


Where $\mathrm{w}$ is the weight, $\mathrm{b}$ is the LSSVMR bias and $\varphi(x)$ is the function used to map the input data of LSSVMR to a higher dimensional space (feature space), so it is expected that the nonlinear data in the input LSSVMR will be linear in the feature space. Equation (9) can be solved by an optimization function to find the minimum value of weight and bias. The LSSVMR optimization function can be written as :

$$
\min _{w, e} J(w, e)=0.5 w^{T} w+\gamma \sum_{x=1}^{n} e_{x}^{2}
$$

Subject to :

$$
y_{x}=w^{T} \varphi\left(x_{x}\right)+e_{x}+b \quad \mathrm{x}=1,2, \ldots, n
$$

Where $e$ is the slack variable and $\gamma$ is the regulator parameter. The LSSVMR optimization function in equation (10) can be solved by Lagrange multiplication, which is formulated by the following equation:

$$
L(b, e, w, \alpha)=J(e, w)-\sum_{x=1}^{n} \alpha_{x}\left\{w^{T} \varphi\left(x_{x}\right)+e_{x}+b-y_{x}\right\}
$$

Where $\alpha$ is the lagrange multiplication parameter. The optimal conditions in the Lagrange equation above can be solved by finding the minimum value of weight, bias and slack parameters and the optimum value of the Lagrange multiplication parameter. This can be resolved under the provisions of Karush Kuhn Tucker (KKT), which can be written as follows:

$$
\left\{\begin{array}{lrl}
\frac{\partial L}{\partial b}=0 \rightarrow & \sum_{x=1}^{n} \alpha_{x}=1 \\
\frac{\partial L}{\partial W}=0 \rightarrow & W=\sum_{x=1}^{n} \alpha_{x} \varphi\left(x_{x}\right) \\
\frac{\partial L}{\partial e_{x}}=0 \rightarrow & \alpha_{x}=\gamma e_{x} \\
\frac{\partial L}{\partial \alpha_{x}}=0 \rightarrow W^{T} \varphi\left(x_{x}\right)+e_{x}+b=0
\end{array}\right.
$$

If the weight and slack variables are eliminated, equation (13) can be formed into the following linear equation:

$$
\begin{aligned}
& {\left[\begin{array}{cc}
0 & \overrightarrow{1}^{T} \\
\overrightarrow{1} & \Omega+\gamma^{-1} I
\end{array}\right]\left[\begin{array}{l}
b \\
\alpha
\end{array}\right]=\left[\begin{array}{l}
0 \\
y
\end{array}\right]} \\
& \Omega_{l j}=\varphi\left(x_{k l}\right)^{T} \varphi\left(X_{j}\right)=k\left(X_{l}, X_{j}\right) \quad l, j=1,2, \ldots, n
\end{aligned}
$$

Where $k\left(x, x_{i}\right)$ is the kernel function. There are several methods that can be used to solve the kernel function. In this paper, the kernel function is solved by using the radial basis function method, which is formulated by:

$$
k\left(X, X_{i}\right)=\exp \left\{-\left\|X-X_{i}\right\|_{2}^{2} / \sigma^{2}\right\}
$$

After solving the kernel function problem, then set :

$$
A=\Omega+\gamma^{-1} I
$$

If $A$ is symmetric, then equation (14) can be solved by:

$$
\begin{aligned}
& \alpha=A^{-1}(y-b \overrightarrow{1}) \\
& b=\frac{\overrightarrow{1}^{T} A^{-1} y}{\overrightarrow{1}^{T} A^{-1} \overrightarrow{1}}
\end{aligned}
$$

After the lagrange multiplication parameters and bias values are obtained through equations (18) and (19), then by substituting the weight parameters in equation (9) into equation (13) and using the Mercer condition in equation ((15), the LSSVMR regression function which is presented in equation (9) can be rewritten as follows:

$$
f(x, w)=y(x)=\sum_{x=1}^{n} \alpha_{x} k\left(x, X_{i}\right)+b
$$

Based on equation (20) it can be concluded that the validity of the LSSVMR algorithm is determined by kernel parameters and bias. This parameter can be obtained through offline supervised training. After the kernel parameters and bias are obtained, then the LSSVMR algorithm is ready to be used for the online MRAS observer.

\section{RESULTS AND DISCUSSION}

The proposed MRAS observer for PMSM was validated through a simulation with Matlab software. The MRAS observer is applied to the PMSM model with a power rating $2.2 \mathrm{~kW}$, pole number 10 , permanent magnetic flux $0.175 \mathrm{~Wb}$, stator resistance $0.2 \mathrm{Ohm}$ and stator inductance $8.3 \mathrm{mH}$.

The simulation was carried out in two stages, namely the offline LSSVMR training stage to obtain both kernel and bias parameters and the online stage 
to test the MRAS observer model with the LSSVMR adaptation mechanism.

LSSVMR offline training is carried out with four inputs and two outputs, as shown in Figure 4. The LSSVMR input is the reference current error in $\alpha \beta$ axis and the estimated current in $\alpha \beta$ axis, while the LSSVMR output is the back emf voltage in $\alpha \beta$ axis. The LSSVMR model used is the LSSVMR algorithm which has been discussed in [10]. The LSSVMR algorithm is trained with 54000 data samples.

LSSVMR algorithm training is carried out by using the radial basis kernel function. The results of this offline training have provided the best kernel functions with values $(262588417,163 ; 0,010325)$. Figure 5 shows the back emf voltage from the LSSVMR training results. Figure 5 (a) shows that the estimated back emf voltage from the LSSVMR algorithm is in accordance with the target value of the LSSVMR training. The LSSVMR training results only provide a maximum error of 0.0025 volts, as shown in Figure 5(b).

These results indicate that the performance of the LSSVMR algorithm is very good in estimating the back emf voltage based on the current value as the input of the LSSVMR. These results also indicate that the LSSVMR algorithm is ready to be tested online as an adaptation mechanism for the MRAS observer.

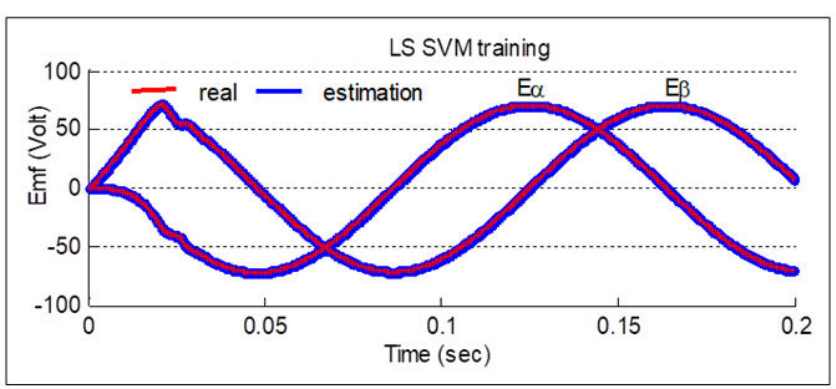

(a)

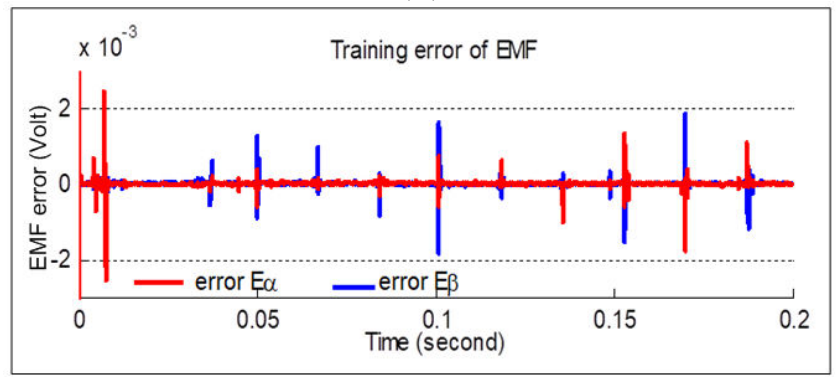

(b)

Gambar 5. The result of LSSVMR training. (a) back emf voltage, (b) error of back emf voltage.

The PMSM rotor position can be obtained based on the estimated back emf voltage by using equation (7). Figure 6 shows the estimated value of the PMSM rotor position based on calculation results.
Figure 6 shows that the estimated value of PMSM rotor position is close to the actual value. This results shows that the adaptation mechanism of MRAS observer by using LSSVMR algorithm has succeeded determining the estimated back emf voltage based on current parameters in the reference PMSM model and the adaptive PMSM model, so that the estimated PMSM rotor position can be close to the actual PMSM rotor position value.

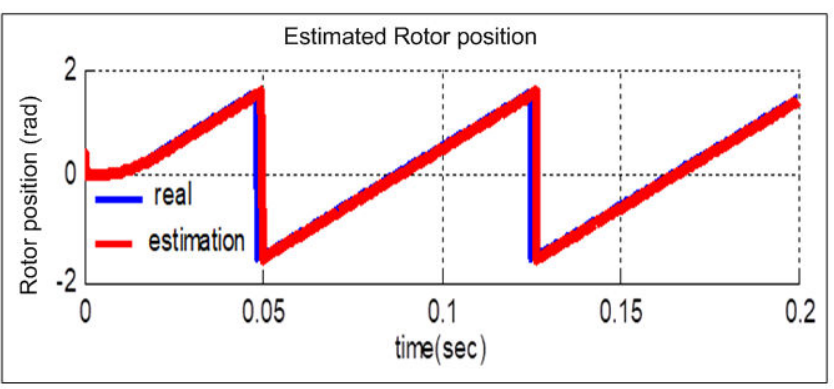

Figure 6. Rotor position

Based on the estimated PMSM rotor position, the estimated PMSM rotaion speed as the MRAS observer output can be calculated by using Equation (8). Figure 7 shows the estimated PMSM rotation speed based on the calculation results.

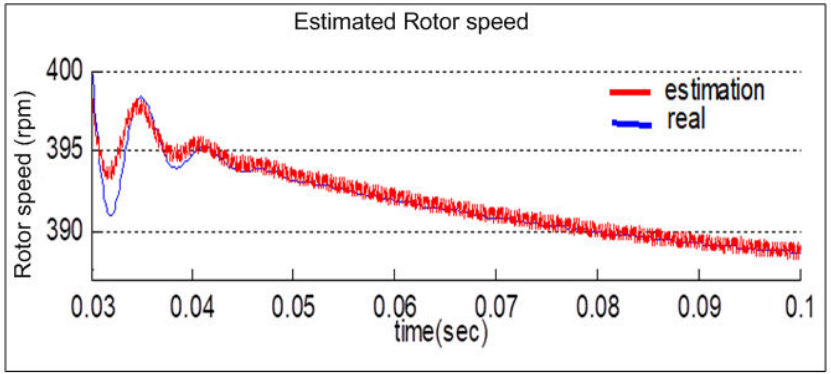

(a)

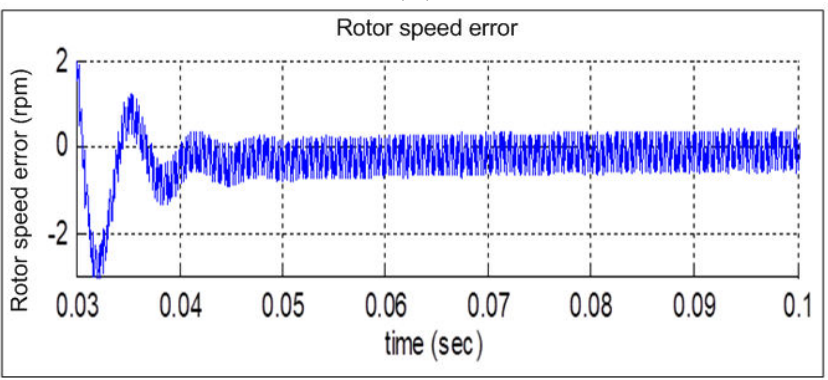

(b)

Figure 7. MRAS observer output. (a) rotor speed (b) rotor speed error

Figure 7(a) shows that the estimated PMSM rotation speed from the MRAS observer has approached the same as the actual value. The maximum error of the PMSM rotation speed from the 
LSSVMR training results is only $2.5 \mathrm{rpm}$, as shown in Figure 7(b). These results indicate that the MRAS observer designed using LSSVMR as an adaptation mechanism has successfully identified the rotor speed according to the actual rotor speed.

After the LSSVMR training for MRAS observers is complete, then the MRAS observers are implemented to the model of PMSM rotation speed control, as shown in Figure 8. This motor speed control system is designed using the Field Oriented Control (FOC) method with a constant electromagnetic torque angle strategy. In this method, the PMSM rotation speed is regulated by controlling the q-axis current of stator, while the daxis current of stator is kept constant at one value. The q-axis stator current regulation is implemented using a Proportional Integral (PI) method. The estimated rotation speed from the MRAS observer is used as the rotation speed feedback for FOC. The rotation PMSM speed is controlled according to the desired reference rotor speed value, as shown in Figure 8.

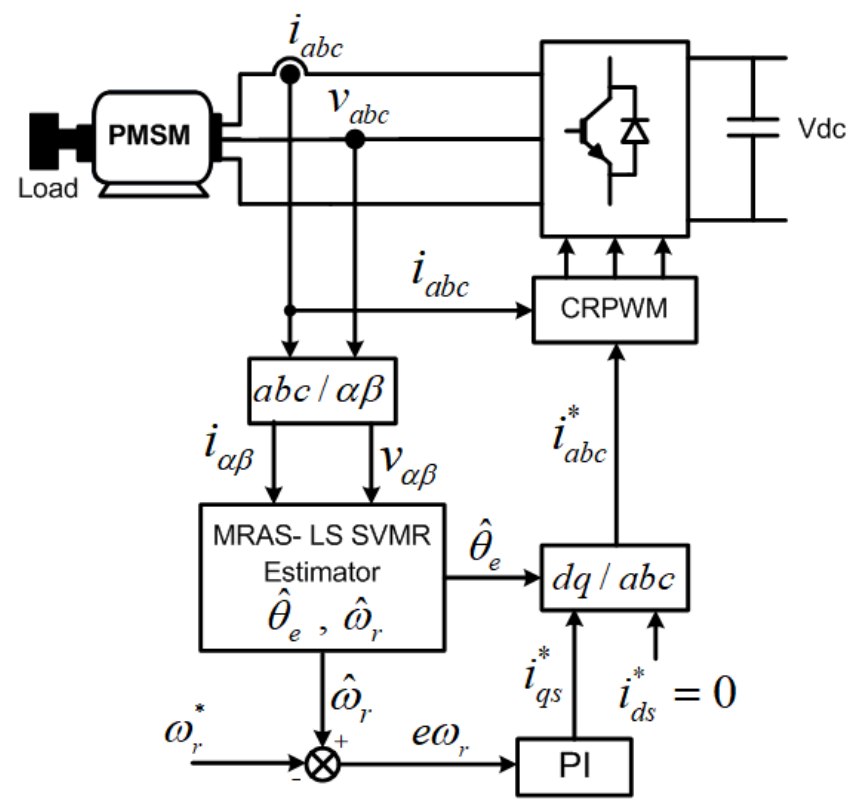

Figure 8. MRAS observer scheme of motor speed control

The proposed MRAS observer based on LSSVMR algorithm is validated through simulation on the model of PMSM rotation speed control.The simulation was carried out with load variation from $5 \mathrm{~N} . \mathrm{m}$ in the initial conditions, then the PMSM load increased to 7 N.m at 0.8 seconds, as shown by the mechanical torque graph in Figure 9.

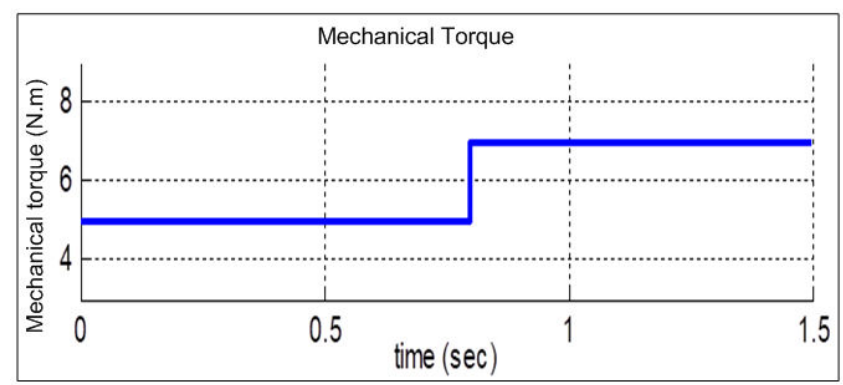

Figure 9. Mechanical torque

The test is done by varying the PMSM speed through the reference speed setting on the control system. The PMSM reference speed is set at $380 \mathrm{rpm}$ in the initial conditions, then increases to $540 \mathrm{rpm}$ at 0.8 seconds, as shown by the rotation speed graph in Figure 10(a).

The simulation results show that the PMSM speed control design with the PI controller-based FOC method using the MRAS observer as the motor speed estimator has successfully controlled the speed according to the reference speed, as shown by the rotation graph in Figure 10(a). The estimated rotation speed from the MRAS observer that presented the actual rotor speed has approached the same as the reference rotor speed. The proposed PMSM speed control system based on MRAS observer only gives a maximum speed error of 3.7 $\mathrm{rpm}$ at transient conditions and close to zero at steady state, as shown the rotor speed graph in Figure 10(b).

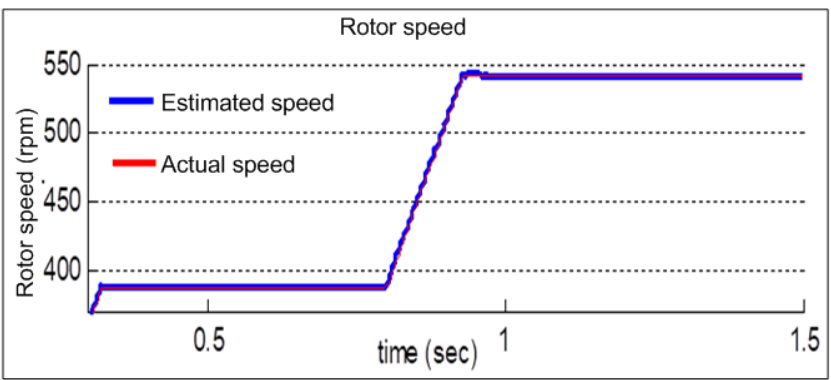

(a)

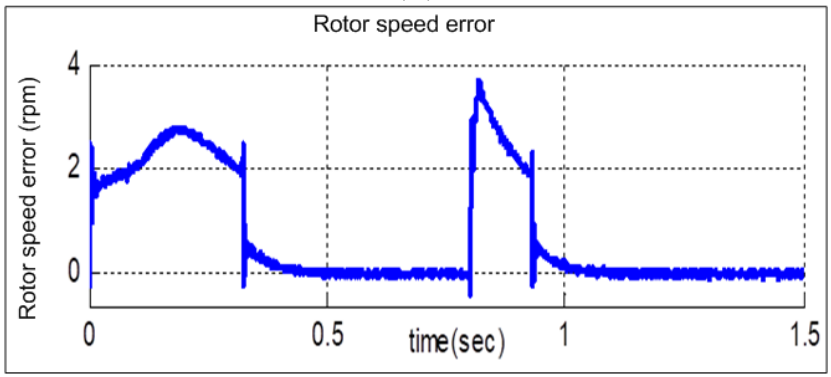

(b)

Figure 10. Simulation results. (a) rotor speed, (b) rotor speed error 
The success of the PMSM rotation speed control system to regulating the rotation speed is inseparable from the success of the MRAS observer in identifying the rotor speed, so that the designed control system can work properly. The validity of the MRAS observer cannot be separated from the reliability of the LSSVMR algorithm in identifying the back voltage emf, so that the PMSM parameters in both the reference PMSM model and the adaptive PMSM model in the MRAS observer have nearly the same value. This makes the estimated rotation speed from the output of MRAS observer follow the actual rotor speed value from PMSM. Figure 11 shows the performance of the MRAS observer based on the LSSVMR algorithm as an adaptation mechanism in the rotation speed control model.

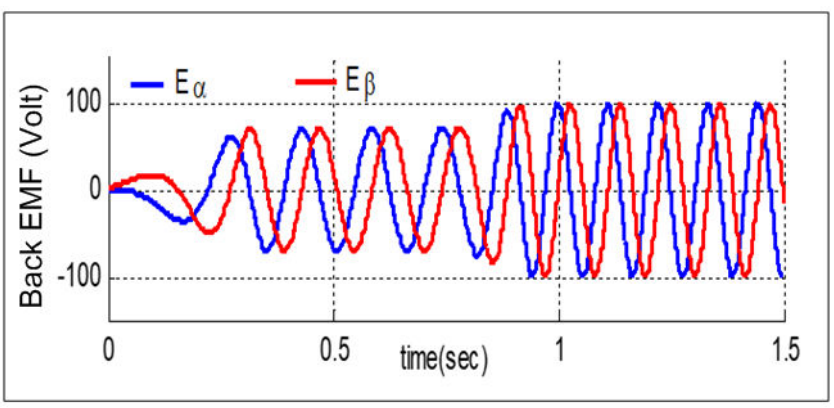

(a)

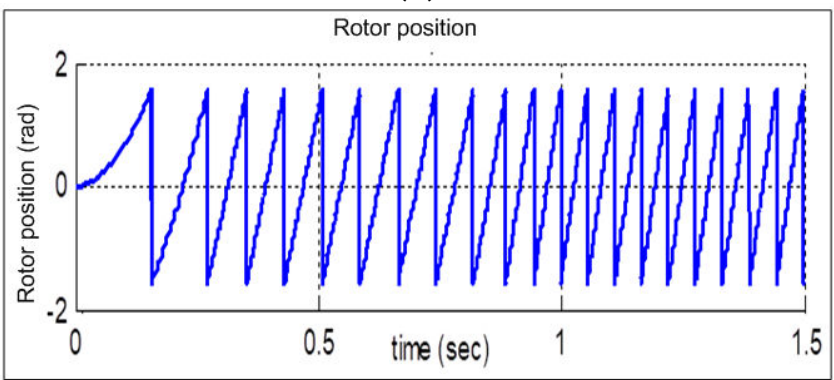

(b)

Figure 11. MRAS observer performances. (a) back emf voltage (b) rotor position

Figure 11(a) shows the estimated value of back emf voltage from LSSVMR algorithm. The waveform of the estimated back emf voltage has produced the estimated PMSM rotor position, as shown in Figure 11(b). The derivative of the estimated rotor position give the estimated rotation speed, as shown in Figure 10 (a), where the estimated rotation speed value has approached the same as the reference speed value. These results indicate that the MRAS observer designed with the LSSVMR algorithm as an adaptation mechanism has successfully identified the PMSM rotation speed in all conditions. This makes the proposed PMSM rotation speed control by using FOC method provide excellent results in controlling the rotor speed in accordance with the reference speed set in the control system.

\section{CONCLUSION}

The motor speed observer is designed to identify motor speed without using a speed sensor. This paper proposes an MRAS observer to identify the PMSM speed. The reliability of the MRAS observer is determined by the adaptation mechanism used. To improve the reliability of the MRAS observer, the LSSVMR algorithm was designed as an adaptation mechanism for the MRAS observer. The MRAS observer based on the LSSVMR algorithm is implemented in the PMSM speed control system. The PMSM speed control system is designed using the PI controller-based FOC method. The simulation results show that the proposed MRAS observer with the LSSVMR algorithm as an adaptation mechanism has successfully identified the motor speed under varying speed and load conditions. The proposed MRAS observer only produces a maximum speed error of $3.7 \mathrm{rpm}$ in transient conditions and close to zero at steady state. This shows that the MRAS observer based on the LSSVMR provides excellent performance in identifying the PMSM rotor speed.

\section{REFERENCES}

[1] M. Yuhendri, Ahyanuardi, and Aswardi, "Direct torque control strategy of PMSM employing ultra sparse matrix converter", International Journal of Power Electronic and Drive System, vol. 9, no. 1, pp. 133-143, 2018.

[2] I.M. Hinkkanen, S.E. Saarakkala, H.A.A Awan, E. Molsa, and T. Tuovinen, "Observers for Sensorless Synchronous Motor Drives: Framework for Design and Analysis," IEEE Transactions on Industry Applications, vol. 54, no. 6, pp. 6090 - 6100, 2018.

[3] T. Shi, Z. Wang, and C. Xia, "Speed Measurement Error Suppression for PMSM Control System Using Self-Adaption Kalman Observer," IEEE Transactions on Industrial Electronics, vol. 62, no. 5, pp. 2753-2763, 2015.

[4] L. Vesely, and Z. Bradac, "MRAS identification of permanent magnet synchronous motor parameters," IFAC Papers Online, vol. 51, no.6, pp. 250-255, 2018

[5] M. Yuhendri, M. Ashari, and M. H. Purnomo, "A novel sensorless MPPT for wind turbine generators using very sparse matrix converter based on hybrid inteligent control," 
International Review of Electrical Engineering, vol. 10, no. 2, pp. 233-243, 2015.

[6] S. Zhang, M. Wang, P. Zheng, G. Qiao, F. Liu, and L. Gan, "An Easy to Implement Hysteresis Model Identification Method Based on Support Vector Regression," IEEE Transactions on Magnetics, vol. 53, no. 11, pp. 1-4, 2017

[7] K. Li, R. Zhang, F. Li, L. Su, H. Wang, and P. Chen, "A New Rotation Machinery Fault Diagnosis Method Based on Deep Structure and Sparse Least Squares Support Vector Machine," IEEE Access, vol. 7, pp. 26571-26580, 2019.

[8] A. Zendehboudi, M.A. Baseer, and R. Saidur, "Application of support vector machine models for forecasting solar and wind energy resources: A review," Journal of Cleaner Production, vol. 199, pp. 272-285, 2018.

[9] S. Nan, L. Sun, B. Chen, Z. Lin, and K.A. Toh, " Density-Dependent Quantized Least Squares Support Vector Machine for Large Data Sets," IEEE Transactions on Neural Networks and Learning Systems, vol. 28, no. 1, pp. 94-106, 2017.

[10] K.D Brabanter,P. Karsmakers, F. Ojeda, C. Alzate, K.D Brabanter, K. Pelckmans, B.D. Moor, J. Vanewalle and J.A.K. Suykens, LS-SVMM lab toolbox user's guide version 1.7. Katholieke Universiteit Leuven, Heverlee, 2010. 